\title{
Audiovisual Speech Web-Lab: An Internet teaching and research laboratory
}

\author{
MICHAEL S. GORDON and LAWRENCE D. ROSENBLUM \\ University of California, Riverside, California
}

\begin{abstract}
Internet resources now enable laboratories to make full-length experiments available on line. A handful of existing web sites offer users the ability to participate in experiments and generate usable data. We have integrated this technology into a web site that also provides full discussion of the theoretical and methodological aspects of the experiments using text and simple interactive demonstrations. The content of the web site (http://www.psych.ucr.edu/avspeech/lab) concerns audiovisual speech perception and its relation to face perception. The site is designed to be useful for users of multiple interests and levels of expertise.
\end{abstract}

Many psychology research laboratories now provide web sites with text and demonstrations describing their research programs. In addition, a handful of internet teaching sites provide full-length psychology experiments on line (e.g., http://www.olemiss.edu/projects/ PsychExps/, http://kahuna.psych.uiuc.edu//ipl/, http://coglab.psych. purdue.edu/coglab/). Yet although these on-line experiment sites allow users to generate analyzable data, they currently provide only cursory descriptions of the conceptual issues behind the experiments (i.e., Francis et al., 2000; McGraw, Tew, \& Williams, 2000). An Internet site that allows users to generate usable data, offers a tutorial on methods, and discusses the theoretical background of the experiments would provide a much more comprehensive introduction to the presented research. We have developed a web site on audiovisual speech perception that combines these components of teaching and research laboratory web sites. The Audiovisual Speech Web-Lab (http://www. psych.ucr.edu/avspeech/lab) provides (1) fully interactive experiments that generate usable data, (2) tutorials on creation of the stimuli and experimental methods, and (3) discussions of the experiments' underlying motivations and theoretical issues all presented at multiple levels of sophistication. The goal of the site is to present an overview of a particular research area using as much handson interactivity as the Internet currently provides.

The web site has been designed for use by individuals with various interests and levels of expertise. A novice user can explore simple demonstrations and summary discussions as well as take a pictorial tour of our laboratory. Intermediate students can access more detailed discussions of relevant theories and related research areas. The on-line experiments and data output can be used for undergraduate laboratories. Advanced users with extensive research

Correspondence should be addressed to L. D. Rosenblum, Department of Psychology, University of California, Riverside, CA 92521 (e-mail: rosenblu@ citrus.ucr.edu). experience and/or familiarity with the research area can access stimuli, data sets, and written reports from the laboratory. Advanced users can also use the on-line experiments to run subjects for their own research purposes. For all users, applications of the research for clinical and industrial settings are discussed. The web site organizes the multiple levels of pedagogy hierarchically to encourage novice users to explore the concepts at increasing levels of sophistication.

The web site demonstrations make use of QuickTime (2000) format movies as well as animated GIFs and graphics. Using dynamic stimuli can provide a much more palpable introduction to the methodological and conceptual issues of a research program (Neuhoff, 2000; Teasley \& Roschelle, 1993). The tutorial text is designed around the graphics and demonstrations. The full-length experiments have been scripted with Authorware (1998) software and have been designed according to the format established for the Psych Experiments web site (McGraw et al., 2000). To accommodate potential researchers, Authorware provides a framework in which scripts can be easily adjusted to vary the number of trials and types of trial used. Users are invited to contact the current designers to guide the development of projects and experimental content.

The projects currently available on the web site will be discussed in turn with regard to their content and development.

\section{The McGurk Effect}

The McGurk effect (Green, 1998; McGurk \& MacDonald, 1976) pairs discrepant auditory and visual speech syllables (e.g., auditory /ba/; visual/va/) to test crossmodal influences on speech perception. These discrepant audiovisual syllables often produce "heard" percepts that are strongly influenced by the visual component (e.g., auditory /ba/ + visual /va/ = "heard" percept /va/ or /fa/). The effect provides a compelling demonstration of how every listener integrates visual speech (lipread) information regardless of the level of hearing. Simple dem- 
onstrations of the McGurk effect are currently available on the Internet (e.g., at http://mambo.ucsc.edu/psl/dwmdir/ da.html, http://kahuna.psych.uiuc.edu//ipl/aud/mcgurk/ level_3.html). However, in order to provide a more extensive understanding of the McGurk effect, we have incorporated several varieties of audiovisual syllables into a full experiment for on-line users. Users are able to select various design aspects of the experiment, participate as subjects, and then survey and analyze the collected data. The stimuli are composed of audiovisual or audio-only speech syllables presented as QuickTime movies. Following each stimulus presentation, observers are presented with six multiple-choice buttons from which they select the option that best describes what they have heard (Green, 1998). Authorware software navigates the user randomly through the different trial types and records whether the correct response has been selected. Upon completion of the experiment, a printable page is generated, displaying a table of the responses for each of the trial types. In addition, users can download data in a more raw format readable by a number of spreadsheet programs (Williams, McGraw, \& Tew, 1999).

The web site also offers a tutorial/demonstration page for the McGurk effect. This page is designed as an introduction to the methods, reported results, and theoretical issues behind the effect. The page guides the user through a simple demonstration, various exercises, and facts relevant to the effect (e.g., its scope). This user-friendly format is intended for more introductory users. From this demonstration page, users are able to open pages discussing the implications of the McGurk effect for theories of perception, neuropsychology, and clinical and industrial applications. Additional pages provide a list of relevant references and links to related web sites.

\section{Lipreading From Moving Dots}

Another section of the web site demonstrates how visual speech can be perceived from faces that are reduced to moving dots (point-light faces). These demonstrations show that isolated dynamic visual speech information, devoid of facial features, is sufficient for visual speech perception (e.g., Rosenblum, Johnson, \& Saldaña, 1996; Rosenblum \& Saldaña, 1996). In one web-site demonstration, users are presented with a point-light face articulating a sentence. The face is then dubbed with the same auditory sentence presented in masking white noise. Users are asked to listen to the sentence with and without viewing the face in order to experience the enhancing effect of the point-lightinformation. Different patterns of pointlights are shown on the face so that users can explore which visible articulatory movements are most enhancing.

The second point-light demonstration implements the McGurk effect (McGurk \& MacDonald, 1976) to show how isolated dynamic visual speech information can be integrated with auditory speech (Rosenblum \& Saldaña, 1996). A full-length Authorware experiment has been developed in which users judge discrepant audiovisual syllables in the manner described above. For this exper- iment, however, the visual stimuli are composed of pointlight faces. Users are allowed to select from different experiment lengths, and data is provided in both tabulated and raw formats.

Additional web pages provide descriptions and photographs of the point-light application procedure. Implications of the point-light technique for telecommunications and determining salient visual speech information are also discussed. More sophisticated theoretical issues are also addressed, such as the relative salience of time-varying visual and auditory speech information. Links to other laboratories using point-light methodologies are provided.

\section{Lipreading From Inverted Lips}

A third section of the web site addresses the relationship between visual speech and face perception. This issue has important implications for theories of speech and face recognition, as well as for modularity (Fodor, 1983) and neuropsychology. For these purposes, a demonstration is implemented which combines the McGurk effect with a well-known phenomenon in the face perception literature known as the Margaret Thatcher effect (Rosenblum, Yakel, \& Green, 2000). The Margaret Thatcher effect involves a facial image in which the eyes and mouth have been inverted (Thompson, 1980). This distortion is much easier to notice when the face is shown upright rather than upside down. In this sense, the Margaret Thatcher effect adds to the literature suggesting that face perception is especially attuned to upright versus inverted faces.

To show that visual speech perception is also influenced by upright face information, a McGurk effect methodology is applied to articulating faces with inverted mouths (Rosenblum et al., 2000). Stimuli are composed of audio / ba/ combined with visual /va/ (usually perceived as "va"), and the visual stimuli show an (articulating) upright face, an inverted face, an inverted face with upright mouth, an upright face with inverted mouth, an isolated mouth shown upright, and an isolated mouth shown inverted. All of these stimuli except for the upright face with inverted mouth induce a McGurk effect. This phenomenon suggests that visual speech perception, like face perception, makes special use of the upright face context.

The web site provides these stimuli in both fullexperiment and demonstration formats. For the full experiment, an Authorware script has been developed to present the stimuli, in both audiovisual congruent and incongruent conditions. Subjects are asked to respond in the manner described above, and both raw and tabulated data are provided on completion of the experiment.

The demonstration version provides full descriptions of the stimuli and their production, along with coverage of the relevant conceptual issues for visual speech and face perception. Animated GIFs of the Margaret Thatcher effect are included and are available to download. For more sophisticated users, issues of modularity and neuropsychology are discussed. Stimuli and research reports are made available, and links to face perception web sites are provided. 


\section{Conclusions}

We provide this web site as both an entry into a particular research area (audiovisual speech perception), as well as a possible prototype web-based teaching tool. We encourage other researchers to consider integrating fulllength on-line experiments into their laboratory web sites. The large on-line community formed around sites which currently provide on-line experiments (e.g., Williams et al., 1999) suggests the appeal of this level of interactivity. By offering on-line demonstrations, tutorials, research reports, as well as full experiments with usable data, laboratory sites could benefit users with a wide variety of interests and backgrounds.

\section{REFERENCES}

Authorware 4.o [Computer software]. (1998). San Francisco: Macromedia.

FoDOR, J. A. (1983). Modularity of mind. Cambridge, MA: MIT Press, Bradford Books.

Francis, G., Neath, I., Stevenson, A. K., Surprenant, A. M., Kim, H., Kelley, M. R., \& Marble, J. (2000). CogLab Online Laboratory [On line]. Available: http://coglab.psych.purdue.edu/coglab/index.html GREEN, K. P. (1998). The use of auditory and visual information during phonetic processing: Implications for theories of speech perception. In R. Campbell, B. Dodd, \& D. Burnham (Eds.), Hearing by eye II: Advances in the psychology of speechreading and auditory-visual speech (pp. 3-25). Hove, U.K.: Psychology Press.
McGraw, K., Tew, M. D., \& Williams, J. E. (2000). Psych experiments: Psychology experiments on the Internet [On line]. Available: http://www.olemiss.edu/projects/PsychExps

McGurk, H., \& MacDonald, J. W. (1976). Hearing lips and seeing voices. Nature, 264, 746-748.

NeUHofF, J. (2000). Classroom demonstrations in perception and cognition using presentation software. Teaching of Psychology, 27, 142-144.

QuickTime5.0 [Computer software]. (2000). Cupertino, CA: Apple Computer.

Rosenblum, L. D., Johnson, J. A., \& Saldaña, H. M. (1996). Point-light facial displays enhance speech in noise. Journal of Speech \& Hearing Research, 39, 1159-1170.

Rosenblum, L. D., \& SALDAÑA, H. M. (1996). An audiovisual test of kinematic primitives for visual speech perception. Journal of Experimental Psychology: Human Perception \& Performance, 22, 318-331.

Rosenblum, L. D., Yakel, D. A., \& Green, K. P. (2000). Face and mouth inversion effects on visual and audiovisual speech perception. Journal of Experimental Psychology: Human Perception \& Performance, 26, 806-819.

Teasley, S. D., \& Roschelle, J. (1993). Constructing a joint problem space: The computer as a tool for sharing knowledge. In S. P. Lajoie \& S. J. Derry (Eds.), Computers as cognitive tools (pp. 229-258). Hillsdale, NJ: Erlbaum.

Thompson, P. (1980). Margaret Thatcher: A new illusion. Perception, 9, 483-484.

Williams, J. E., McGraw, K. O., \& Tew, M. D. (1999). Undergraduate labs and computers: The case for PsychExps. Behavior Research Methods, Instruments \& Computers, 31, 287-291.

(Manuscript received November 27, 2000; accepted for publication March 13, 2001.) 